\title{
Research on Real-time video multiple hops relay transmission in Wireless Ad Hoc Network
}

\author{
Yang Tao, Youfeng Gu \\ School of Communication and Information Engineering, University of Posts and \\ Telecommunications, Chongqing,400065, China
}

Keywords: Ad Hoc, Real-time, Video, Hop

\begin{abstract}
The transmission of real-time video need a stable link and is also very sensitive to delay and packet loss, this is a huge challenge to wireless ad hoc network[1,2,3] which has dynamic network topology and limited bandwidth. In this paper, a method to transmit video streams in multi-hop network is proposed which set a relay node every three hops on the path of each flow. The relay nodes are responsible to repair the video data and then forward the packets to next nodes. This method can improve the video quality in the receiver by increasing the video packet delivery ratio, and even can realize video transmission from one node to several nodes.
\end{abstract}

\section{Introduction}

Although the development of the wireless ad hoc network has been relatively mature. To achieve real-time video streaming transmission of multiple hops[4,5] and make the video data are transmitted to users in the wireless ad hoc network. What is more,it can ensure the quality of video and play in real time.It is also needs to consider the ad hoc network bandwidth limitations of single channel, high packet loss rate, link instability and so on.This can cause the decrease of the quality of the video.

Using suitable for the narrow bandwidth of the routing protocol of wireless network environment[6],the video data after three jump of packet loss rate is only about five over one thousand.We can take advantage of the lost packet retransmission method to repair the video data. After repairing the video data ,it is the same as the original video data that video source node sends.It can also maintain a relatively low packet loss rate after three jump transmission.In theory, video data can keep high PDR (packet delivery ratio)after the transmission of infinite jump.In the multiple hops transmission process,video playback quality problems caused by the lost packets.The user node can receive and smooth play high quality video.But,the proposed method enables user node can receive and smooth play high quality video.

\section{Multiple hops relay mechanism}

\section{The real-time video transmission method}

In order to ensure the video data transmission are not produce network congestion[7], and reduce the transmission delay in the network.In front of the video connections, we also need to find a link to satisfy the need of video transmission minimum bandwidth. If the minimum bandwidth for Bn of video transmission in the wireless ad hoc network. So all nodes of the available bandwidth on the link must be greater than the Bn.In front of the user node to send video request, we need to check whether its own bandwidth available to meet receiving video data.If not satisfied, end the video request process directly.

User node requests video transmission process can be divided into the following steps:

STEP1: User node checks whether the current available bandwidth is greater than the minimum bandwidth requirements of video transmission Bn.If more than, broadcast video request response packet (VREP) to neighbor nodes.If less than, end request process.

STEP2:Node checks address of the packet after receiving video request (VREQ) service packet.If the same as their address, the current node as the service node, then turn to STEP3; If the address exist in their own video service list (VSL), the current node as the relay node,then turn to 
STEP5; If it is different from their own address, and does not exist in the VSL.Therefore,current node for the common routing nodes, and then turn to STEP6.

STEP3:According to the video user table (VUL), service node checks whether there is a service node address as this address table. If more than, sending video request response (VREP) packet to the user node ;If not, turn to STEP4.

STEP4:Checking whether the current available bandwidth is greater than the Bn. If more than, then send video request response (VREP) data to the user node; If less than, then send video request failure (VERR) packets to the user node .

STEP5: Relay node sends video request failure (VERR) packet to the user node.

STEP6:Checking whether the current available bandwidth is greater than the Bn.If more than, turn to STEP7; If less than, discarded VREQ packet.

STEP7:Comparing the current available bandwidth and packet minimum bandwidth of the link.If less than, updating VREQ packet minimum bandwidth of the link for the current available bandwidth and broadcast to the neighbor nodes.

STEP8:User node receives video request failed (VERR) packet and end request process.

STEP9:User node receives video request response (VREP) packet after waiting for a response time point of the reaches the preset, it is means the request process is complete.

The process of establish relay

After waiting for a response time, user node receive VREP packet to process.It reads the link information in the packet.According to the formula of link cost, it chooses the minimum cost of a transmission link and sends connection confirmation message (LACK) to the corresponding service node or relay node. The price of the link can be through the following formula:

$$
\Gamma_{\text {cost }}=C_{1} * \frac{N_{1}-N_{2}-1}{3} * M+C_{2} * N_{2} *\left(\frac{B_{n}}{B_{\min }}\right)+C_{3} * \frac{N_{2}-1}{3} * M
$$

Among them, N1 represents hop to a service node (SN). N2 represents hop to a user node(UN). ${ }^{B_{\min }}$ represents the minimum bandwidth of the link. $B_{n}$ is the minimum bandwidth demand for video transmission. $\mathrm{M}$ as the price of video data is processed by the relay node. $\mathrm{C} 1, \mathrm{C} 2$ and $\mathrm{C} 3$ are impact factor, they satisfy the formula: $1+\mathrm{C} 2+\mathrm{C} 3=1$.

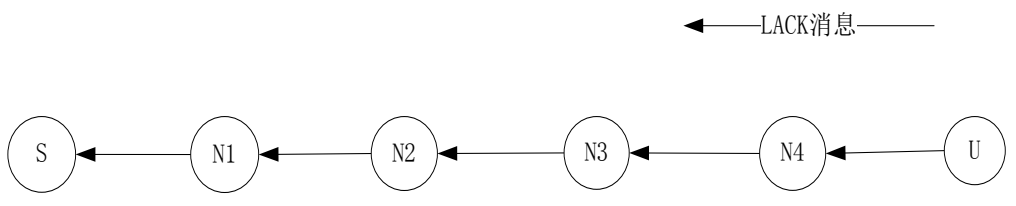

Fig. 1 The process of establish relay

User node U generates LACK data packets, the hop count is five.The RN address for the node U's address, SN and destination node address are node S's address. When N4 receive LACK packet,it checks the hop count and judges themselves for the common routing node.The node S's address is placed into the VUL table of SN address. Node S's address is placed into the VUL as the next RN address. When N3 receive LACK packet and the hop count is 3,N3 will become a relay node.The node S's address and hop are placed into VSL (video service list) list of SN address and hop count to SN.What is more ,the address of node $S$ and U are placed into VUL (video user list) and update current RN LACK packet address for node N3 then continue forward. When the node S receive LACK data packets, the address of the node S and N3 as the VUL list items.

\section{The working process of the relay}

In the process of video relay, source node starts using established video transmission link to send video data to user nodes. Video packet contains the SN address and packet number in Baotou. According to the VSL,node can judge whether the node is the relay node. If the node is a relay node, then the video data are cached, repaired and generated video data packets. If it is not a relay nodes, but its VUL video data packets in SN address then forwarding video data packets directly. The above two conditions are not met, then discarding the video data packets directly. VSL also contains user nodes and service nodes information. Then the video data are cached, repaired when the user receive video data packet.Therefore, user node is corresponding to the service node of relay 
nodes.According to the user id in the VUL ,node judges whether play video data.

\section{Simulation}

\section{Simulation Environment}

Between two nodes are far apart, we compare the two cases.One kind is with multiple hops relay mechanism, another without multiple hops relay mechanism. By comparing the changes of packet delivery rate , average end-to-end delay with the change of time, the average packet delivery rate and the changes of the average end-to-end delay in the one-to-many transmission when video transmission with the increase in the number of hop.

Setting in the area of $1000 \mathrm{~m} * 1000 \mathrm{~m}$, the random distribution of 32 nodes and each node of the communication range is set to $250 \mathrm{~m}$. Node adopts IEEE802.11 media access control and the channel bit rate of 2 Mbps. What is more,each node adopts the AODV[8,9] routing protocol. Simulation use random waypoint mobility model, the maximum rate of mobile node $10 \mathrm{~m} / \mathrm{s}$ and the simulation time is set to $900 \mathrm{~s}$. Table 1 shows the basic simulation environment parameters.

Table1 Basic simulation environment parameters

\begin{tabular}{|c|c|}
\hline The simulation parameters & The parameter value \\
\hline Routing protocol & AODV \\
\hline Area (m2) & $1000 \times 1000$ \\
\hline Number of nodes & 32 \\
\hline Transmission range (m) & 250 \\
\hline The bandwidth (Mbps) & 2 \\
\hline The MAC layer protocol & IEEE802.11 \\
\hline Mobile model & mandom waypoint mobility \\
\hline Node movement speed (m/s) & $0 ~ 10$ \\
\hline Data packet length (Byte) & 512 \\
\hline Simulation time(s) & 900 \\
\hline
\end{tabular}

\section{Simulation Result}

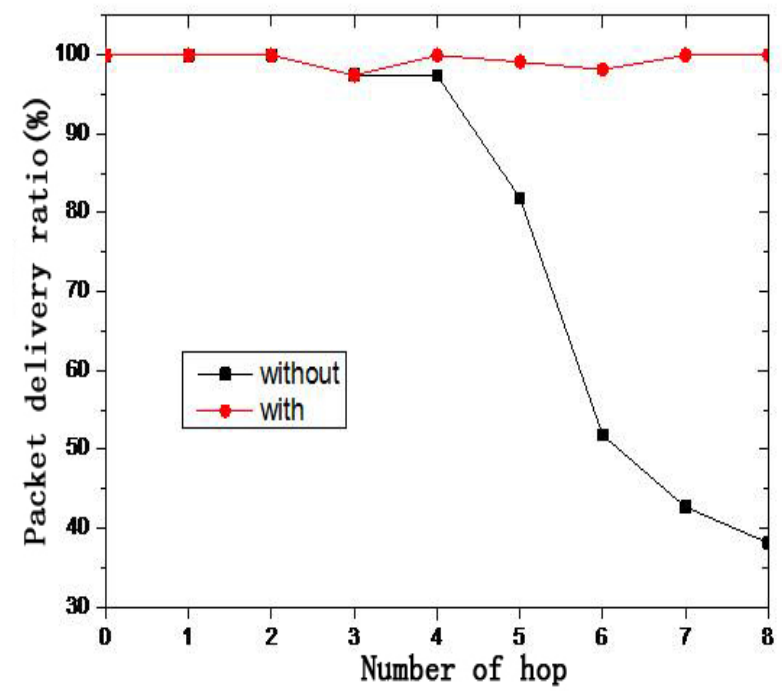

Fig.2 Packet delivery ratio of one-to-one transmission

It can be seen from fig.2, packet delivery rate begin to drop dramatically with no multiple hops 
relay mechanism in the hop count is greater than 4.And the packet delivery rate has less than $50 \%$ in the six hop.Every three jump set a relay node on the transmission path with multiple hops relay mechanism. The relay node repairs the video data, so that the packet delivery rate has been maintained at a higher level, and effective guarantee the quality of video transmission.

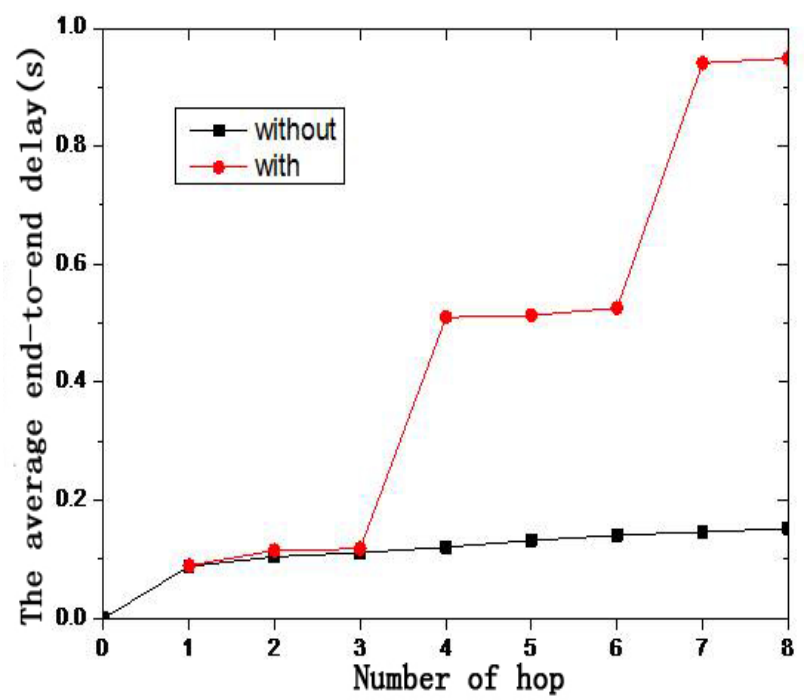

Fig.3 Average end-to-end delay of one-to-one transmission

It can be seen from fig.3,the average end-to-end delay is bigger with multiple hops relay mechanism than without multiple hops relay mechanism.Becase the relay node repairs video data packet,it adds transmission delay. But this is an acceptable scope, the users only need to increase the buffer time in front of the video playback.It is not affect the fluency of video playback.

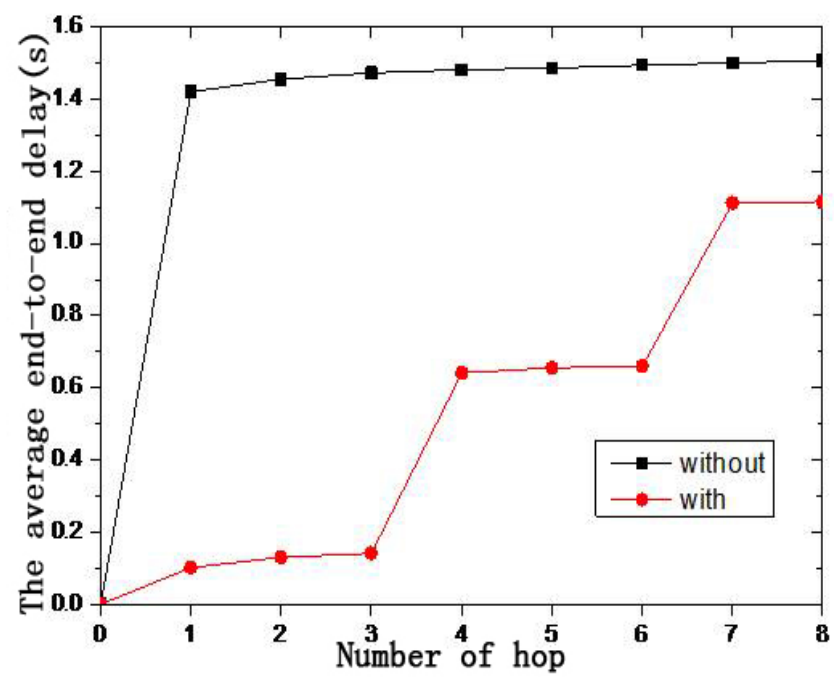

Fig.4 The average packet delivery ratio of one-to-many transmission

Compared with one-to-one transmission in the fig.4, the average packet delivery rate is same with multiple hops relay transmission. In the case of multiple hops relay mechanism, the average packet delivery rate decline faster with the increase of hop.Becauses multiple hops relay transmission takes full advantage of the broadcast characteristic of the node and improve the bandwidth utilization.It can guarantee a higher rate of delivery.In the environment of the limited bandwidth,the one-to-many video transmission will be affected by the node processing power and bandwidth without multiple hops relay mechanism.It is unable to effectively guarantee the video data packet delivery ratio. 


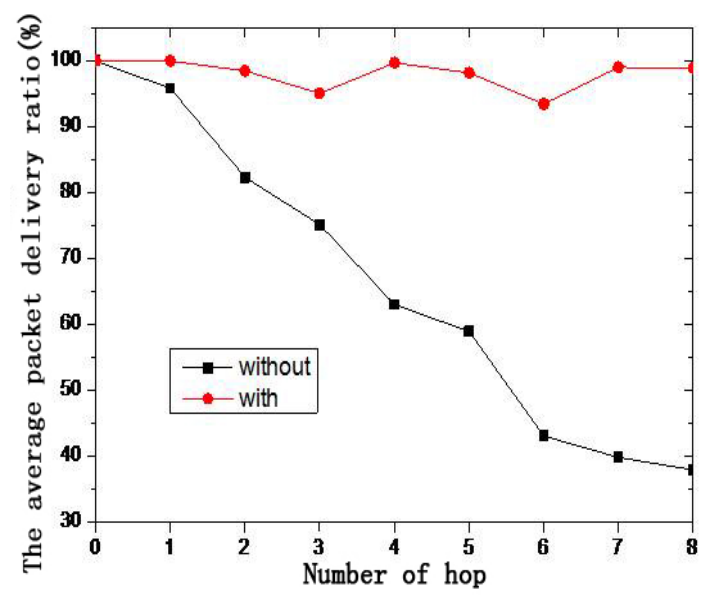

Fig.5 Average end-to-end delay of one-to-many transmission

It can be seen from fig.5, the average end-to-end delay is much smaller with multiple hops relay mechanism than without it.In the case of multiple hops relay mechanism, a node needs to send 5 video streams at the same time in one-to-many transmission. Therefore, it causes the video stream end-to-end delay are significantly increased .Of course,it also increase the average end-to-end delay.

\section{Conclusion:}

Using NS2 simulation platform in this paper, we can analysis the performance of proposed real-time video multiple hops relay mechanism. The simulation results show that the real-time video multiple hops relay transmission method can effectively guarantee the quality of video data transmission through multiple hops, and can realize the one-to-many video transmission.

\section{References}

[1]Xiao Hannan, W. K. G. Seah, Kee Chaing Chua. A flexible quality of service model for mobile ad-hoc networks[C]. 2000 IEEE 51st Vehicular Technology Conference Proceedings,pp.445-449, 2000.

[2]Xiao Hannan, Kee Chaing Chua, W. Seah, A. Lo. On service prioritization in mobile ad-hoc networks[C]. IEEE International Conference on Communications,pp.1900-1904, 2001.

[3]Gahng-Seop Ahn, A. T. Campbell, A. Veres, Li-Hsiang Sun. SWAN:service differentiation in stateless wireless ad hoc networks[C]. Twenty-First Annual Joint Conference of the IEEE Computer and Communications Societies,pp.457-466, 2002.

[4]Zhao Yu-ting, Dai Guan-zhong, Mu De-jun. Performance Evaluation of Real-Time Video over Wireless Ad Hoc Networks[C]. Seventh International Conference on Grid and Cooperative Computing,pp.653-657, 2008.

[5]Lu Qin, Du Liebo, Zuo Zhen, Xiao Xuemin. Improved Multi-Path AODV Protocols for Real-Time Video Transport over Mobile Ad Hoc Networks[C]. Pacific-Asia Workshop on Computational Intelligence and Industrial Application,pp.621-625, 2008.

[6]M.Sivajothi, Dr. E. R. Naganathan. An Ant Colony Based Routing Protocol to Support Multimedia Communication in Ad Hoc Wireless Networks. International Journal of Computer Science and Network Security[J],8(7),pp.100-105, 2008.

[7]Xu Changbiao, Long Keping, Yang Shizhong.Dual AIMD-Based TCP Congestion Control[J].Journal of computer research and development, 40(8),pp.1175-1180, 2003.

[8]Ram S. Dahal, Teerapat Sanguankotchakorn. QoS routing in MANET through Cross-Layer Design with BER and modifying AODV[C], 2011Second Asian Himalayas International Conference on Internet(AH-ICI), pp.1-4,2011.

[9]Hua YANG and Zhi-yuan LI, Simulation and Anlysis of a Modified AODV Routing Protocols,Computer Science and Network Technology (ICCSNT), pp.1440-1444, 2011. 